\title{
Measures of Decoherence
}

\author{
Leonid Fedichkin, Arkady Fedorov and Vladimir Privman
}

\author{
November 2, 2018 \\ Center for Quantum Device Technology, \\ Department of Physics and \\ Department of Electrical and Computer Engineering, \\ Clarkson University, Potsdam, New York 13699-5720
}

\begin{abstract}
Methods for quantifying environmentally induced decoherence in quantum systems are investigated. We formulate criteria for measuring the degree of decoherence and consider several representative examples, including a spin interacting with the modes of a bosonic, e.g., phonon, bath. We formulate an approach based on the operator norm measuring the deviation of the actual density matrix from the ideal one which would describe the system without environmental interactions.
\end{abstract}

Keywords: decoherence, environment, quantum computing, relaxation, spin, thermalization

Citation: Proc. SPIE $\underline{5105}, 243-254$ (2003)

E-print: cond-mat/0303158 at www.arxiv.org

\section{Introduction}

Evolution of a quantum system exposed to an environment is described by the density matrix and deviates from the ideal, usually pure-state, dynamics. In this work, we consider the problem of quantifying this deviation by single numerical measures, derived from the full set 
of the density matrix elements. Depending on their strength and nature, environmental interactions can lead to various relaxation and even measurement-type processes. We will use the term "decoherence" generically. Our focus will be on the environmental effects that represent "noise" and cause small deviations from the desired isolated-system dynamics. Establishment of the threshold criteria for fault-tolerant quantum computation, ${ }^{1}$ has made estimation of the noise due to the environmental effects important for evaluating quantum computing systems.

Superposition of quantum states is crucial in utilizing quantum parallelism for quantum computation. Therefore quantum algorithms usually deal with pure or nearly pure states. There are actually two possible measures of decoherence: deviation from $a$ pure state, and deviation from a particular pure state, or nearly-pure mixed state, which can be time-dependent. Even if the state of the system remains pure, it might deviate from the dynamics desired for a particular controlled quantum process. The ultimate goal of the studies of the type reported here would be to identify simple numerical measures of degree of decoherence occurring during the "clock" times of quantum computing gate functions, to compare with the fault-tolerance requirements in various quantum error correction schemes.

Let us assume that a quantum system of interest, denoted by $S$, is prepared at time $t=0$ in some pure quantum state $|\varphi\rangle$. At times $t>0$, the system can be subject to noise, i.e., coupling to the modes of the environment, but also to "controlling" interactions required for quantum computing. Here we consider a system only exposed to the environmental noise, because the degree of decoherence can be usually approximately evaluated for time scales shorter than or comparable to the times of quantum control. The quantum system can then be described by the reduced density operator, $\rho(t)$, which can be obtained from the overall density operator by tracing over all the environmental degrees of freedom.

For the initial state, the density matrix has the form $\rho(0)=|\varphi\rangle\langle\varphi|$. The interaction with the surrounding, which is usually assumed to be a large macroscopic system in thermal equilibrium at temperature $T$, leads to thermalization of the quantum system. The reduced density matrix of the system for large times should approach

$$
\rho \rightarrow e^{-\beta H_{S}} / \operatorname{Tr}_{S}\left(e^{-\beta H_{S}}\right), \quad \text { as } t \rightarrow \infty,
$$

where $\beta=1 / k T$. Here $H_{S}$ is the Hamiltonian of the system. In the energy eigenbasis, $\left|\phi_{n}\right\rangle$, the density matrix elements behave as follows,

$$
\begin{gathered}
\rho_{m n}=\left\langle\phi_{m}|\rho| \phi_{n}\right\rangle \rightarrow 0, \quad \text { as } t \rightarrow \infty \quad(m \neq n), \\
\rho_{n n}=\left\langle\phi_{n}|\rho| \phi_{n}\right\rangle \rightarrow e^{-\beta E_{n}} / \sum_{k} e^{-\beta E_{k}}, \quad \text { as } t \rightarrow \infty .
\end{gathered}
$$

Usually, thermalization sets in as the slowest relaxation process, and its description requires a phenomenological Markovian assumption..$^{2-4}$ 
In this work, we consider several approaches to measuring the degree of decoherence due to interactions with the environment. In Section 2, we discuss the approach based on the asymptotic relaxation time scales. The entropy and idempotency-defect measures are addressed in Section 3. The fidelity measure of decoherence is discussed in Section 4. Next, in Section 5, we present our results on the operator norm measures of decoherence. Section 6 presents a discussion of an approach to eliminate the initial-state dependence of the decoherence measures, as well as of extensivity properties for multiqubit systems.

\section{Relaxation Time Scales}

Markovian approximation schemes typically yield exponential approach to the limiting values of the density matrix elements for large times. ${ }^{2-4}$ For the two-state system, this defines the thermalization time scales $T_{1}$ and $T_{2}$, associated, respectively, with the approach by the diagonal (thermalization) and off-diagonal (dephasing, decoherence) density-matrix elements to their limiting values; see $(2,3)$. More generally, for large times we expect

$$
\begin{gathered}
\rho_{n n}(t)-\rho_{n n}(\infty) \propto e^{-t / T_{n n}}, \\
\rho_{n m}(t) \propto e^{-t / T_{n m}} \quad(n \neq m) .
\end{gathered}
$$

The shortest time among $T_{n n}$ is often identified as $T_{1}$. Similarly, $T_{2}$ can be defined as the shortest time among $T_{n \neq m}$. These definitions yield the characteristic times of thermalization and decoherence.

For decoherence and thermalization times, the following inequality commonly holds, $T_{2} \leq$ $T_{1}{ }^{3}$ though this relation usually does not apply within Markovian approximations. Therefore, the decoherence time is a more crucial parameter for quantum computing considerations. The time scale $T_{2}$ is compared to the "clock" times of quantum control, i.e., the quantum gate functions, $T_{g}$, in order to ensure the fault-tolerant error correction criterion $T_{g} / T_{2} \leq O\left(10^{-4}\right) .^{5}$

The disadvantages of this type of analysis are that the exponential behavior of the density matrix elements in the energy basis is applicable only for large times, whereas for quantum computing applications, the short-time behavior is usually relevant. ${ }^{6}$ Moreover, while the energy basis is natural for large times, the choice of the preferred basis is not obvious for short and intermediate times. ${ }^{6,7}$ Therefore, the time scales $T_{1}$ and $T_{2}$ have limited applicability in evaluating quantum computing error correction criteria. Their obvious advantage is in that of all the measures discussed in this article, they are the only experimentally observable time scales, as long as no multi-qubit quantum computer was built. 


\section{Quantum Entropy}

An alternative approach is to calculate the entropy ${ }^{8}$ of the system,

$$
S(t)=-\operatorname{Tr}(\rho \ln \rho)
$$

or the idempotency defect, called also the first order entropy, ${ }^{9-11}$

$$
s(t)=1-\operatorname{Tr}\left(\rho^{2}\right) .
$$

Both expressions are basis independent, have a minimum at pure states and effectively describe the degree of the state's "purity." Any deviation from the pure state leads to the deviation from the minimal values, 0 , for both measures,

$$
S_{\text {pure state }}(t)=s_{\text {pure state }}(t)=0
$$

Let us consider a simple example of a two-level system in the excited state $|1\rangle$ at $t=0$, which decays to a ground state $|0\rangle$ via the interaction with a reservoir at zero temperature $T=0$, e.g., the spontaneous decay of an atom in vacuum due to the electromagnetic field. Except for very short times, ${ }^{3}$ the dynamics of the system is well described by the following density matrix,

$$
\begin{gathered}
\rho_{11}(t)=e^{-\Gamma t}, \quad \rho_{00}(t)=1-e^{-\Gamma t}, \\
\rho_{01}(t)=\rho_{10}(t)=\rho_{01}(0)=\rho_{10}(0)=0,
\end{gathered}
$$

where $\Gamma$ is the rate of the spontaneous decay.

In Figure 1, we plot the two entropy measures of decoherence as functions of time, for $\Gamma=1$. Both curves illustrate that these measures determine deviations from "purity," but are not sensitive to deviations from any particular pure (or mixed) state. It is likely that entropy measures are useful in situations when the system has evolved little from a particular initial pure state under its own dynamics and environmental influences. Entropy deviations can thus be used to quantify degree of decoherence/relaxation for short times, ${ }^{6}$ when the system is still sufficiently far from any but the ideal pure state (corresponding to evolution under $H_{S}$ only).

One can derive ${ }^{9}$ the short-time perturbative expansion for $s(t)$. Let $R(t)$ denote the density matrix of the system plus environment, and, for definiteness, assume that the system is initially in the pure state $|\psi\rangle$, unentangled with the environmental bath $(B)$ of modes which are described by the density matrix $\theta$. Then,

$$
\rho(t)=\operatorname{Tr}_{B} R(t)
$$




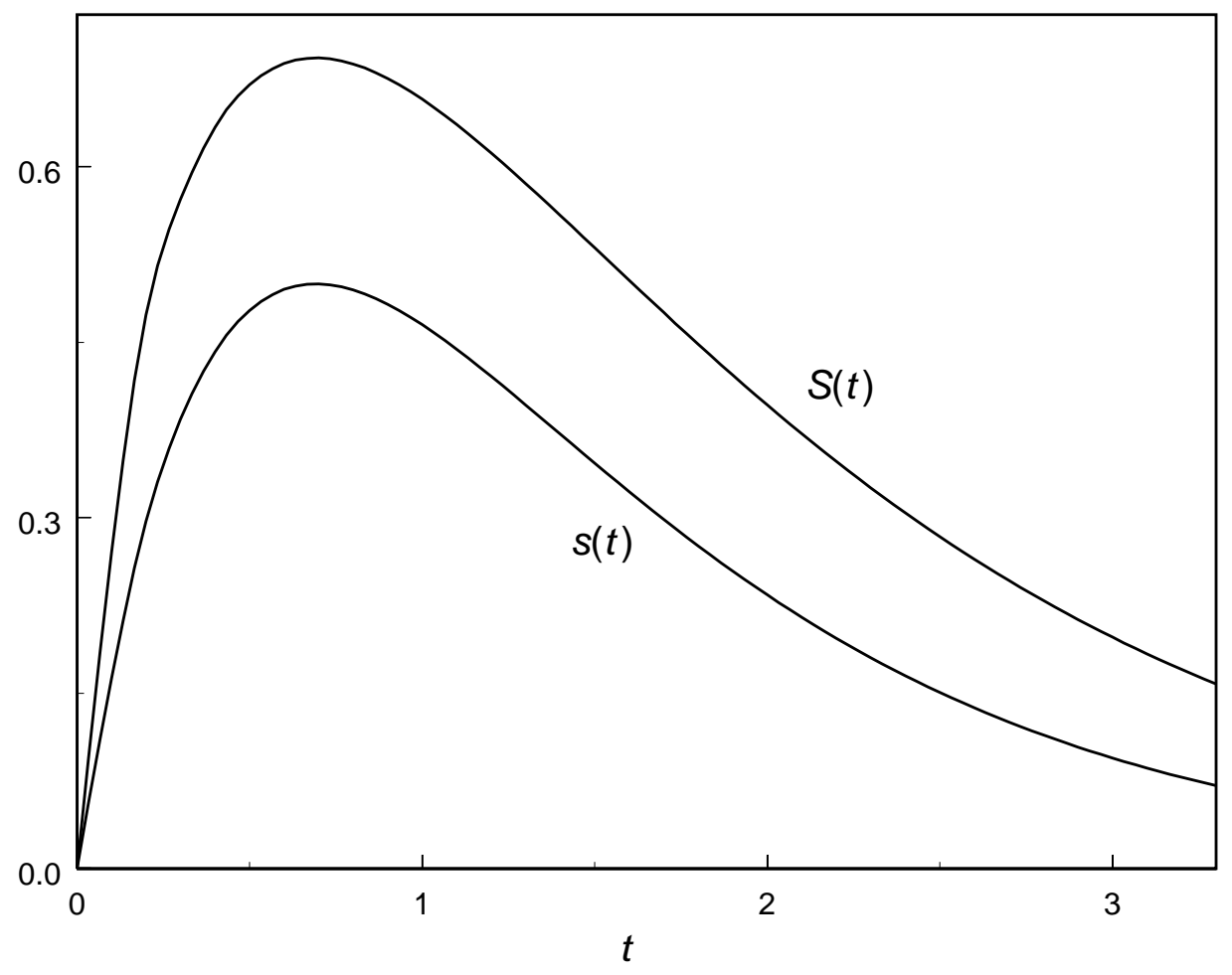

Figure 1: Entropy measures of decoherence for the two-state spontaneous decay model. The upper curve: the full entropy. The lower curve: the first-order entropy.

$$
\begin{gathered}
R(t)=e^{-i H t} R(0) e^{i H t}, \\
R(0)=(|\psi\rangle\langle\psi|) \otimes \theta, \\
H=H_{S}+H_{B}+V,
\end{gathered}
$$

where $H$ is the total Hamiltonian, $H_{S}$ is the Hamiltonian of the quantum system, $H_{B}$ is the Hamiltonian of the environmental bath, $V$ is the interaction Hamiltonian. Here we use the units $\hbar=1$, and we omit the direct product symbol in what follows. We get the expansion of the form

$$
s(t)=\left(t / \tau_{s}\right)^{2}+o\left(t^{2}\right)
$$




$$
1 /\left(2 \tau_{s}^{2}\right)=\left\langle\left(\langle V\rangle_{B}\right)^{2}\right\rangle_{S}+\left\langle\left(\langle V\rangle_{S}\right)^{2}\right\rangle_{B}-\left\langle\left\langle V^{2}\right\rangle_{S}\right\rangle_{B}-\left(\left\langle\langle V\rangle_{S}\right\rangle_{B}\right)^{2}
$$

where $\langle\ldots\rangle_{B},\langle\ldots\rangle_{S}$ denote averaging with respect to $\theta$ and $\rho(0)$, respectively. Here, as usual, average of an operator stands for the trace of its product with the density matrix. Obviously, $\left\langle\langle\ldots\rangle_{S}\right\rangle_{B}=\left\langle\langle\ldots\rangle_{B}\right\rangle_{S}$. The expression (16) is only well defined when all the traces yield final results, which should be usually the case for finite-temperature thermal initial bath density matrix $\theta$, or for projection-operator $\theta$. More sophisticated short-time approximations have been proposed ${ }^{6}$ see below.

For an instructive example, let us consider next a rather general model of the two-level system interacting with a boson-mode reservoir. ${ }^{12}$ As usual, to evaluate the density operator dynamics, we have to use some approximations. For short time scales, we apply the recently developed short-time approximation, ${ }^{6}$ rather than the straightforward perturbative expansion (15). The Hamiltonian of the system has the form,

$$
H=H_{S}+H_{B}+V=-\frac{\Omega}{2} \sigma_{z}+\sum_{k} \omega_{k} a_{k}^{\dagger} a_{k}+\sigma_{x} \sum_{k}\left(g_{k} a_{k}^{\dagger}+g_{k}^{*} a_{k}\right)
$$

Here $H_{S}=-(\Omega / 2) \sigma_{z}$ is the Hamiltonian of the quantum system, $H_{B}=\sum_{k} \omega_{k} a_{k}^{\dagger} a_{k}$ is the Hamiltonian of the bath modes, and the remaining term, $V$, is the interaction Hamiltonian; $a_{k}, a_{k}^{\dagger}$ are the boson annihilation and creation operators; $\sigma_{i}(i=x, y, z)$ are the Pauli matrices; $\Omega>0$ is the energy gap. The eigenstates of $\sigma_{x}$ will be denoted by

$$
\sigma_{x}| \pm\rangle= \pm| \pm\rangle
$$

where $| \pm\rangle=(|0\rangle \pm|1\rangle) / \sqrt{2}$, and $|0\rangle=|\uparrow\rangle,|1\rangle=|\downarrow\rangle$ are the ground and excited states respectively.

Now, to obtain the short time dynamics of the system, one can use the approximation, ${ }^{6}$

$$
\begin{gathered}
\rho_{m n}(t)=\sum_{\substack{p, q=0,1 \\
\mu, \nu= \pm 1}}\left\{e^{i\left(E_{q}+E_{n}-E_{p}-E_{m}\right) t / 2}\langle m \mid \mu\rangle\langle\mu \mid p\rangle\langle q \mid \nu\rangle\langle\nu \mid n\rangle \rho_{p q}(0) e^{-B^{2}(t)\left(\eta_{\mu}-\eta_{\nu}\right)^{2} / 4+i C(t)\left(\eta_{\mu}^{2}-\eta_{\nu}^{2}\right)}\right\} \\
B^{2}(t) \equiv 8 \sum_{k} \frac{\left|g_{k}\right|^{2}}{\omega_{k}^{2}} \sin ^{2} \frac{\omega_{k} t}{2} \operatorname{coth} \frac{\beta \omega_{k}}{2} \\
C(t) \equiv \sum_{k} \frac{\left|g_{k}\right|^{2}}{\omega_{k}^{2}}\left(\omega_{k} t-\sin \omega_{k} t\right)
\end{gathered}
$$


Here the Roman-labeled states, $|i\rangle$, are the eigenstates of $H_{S}$ corresponding to the eigenvalues $E_{i}=(-1)^{(i+1)} \Omega / 2$, with $i=m, n, p, q=0,1$. The Greek-labeled states, $|\zeta\rangle$, are the \pm eigenstates of $\sigma_{x}$ in (18), with eigenvalues $\eta_{\zeta}=\zeta$, where $\zeta=\mu, \nu= \pm 1$. Evaluation of (19) yields the following expressions,

$$
\begin{gathered}
\rho_{11}(t)=\frac{1}{2}\left[1+e^{-B^{2}(t)}\right] \rho_{11}(0)+\frac{1}{2}\left[1-e^{-B^{2}(t)}\right] \rho_{00}(0), \\
\rho_{10}(t)=\frac{1}{2} e^{-i \Omega t}\left[1+e^{-B^{2}(t)}\right] \rho_{10}(0)+\frac{1}{2}\left[1-e^{-B^{2}(t)}\right] \rho_{01}(0) .
\end{gathered}
$$

It transpires that the time-dependence of the density matrix elements within this approximation is not exponential. For estimation of the departure from the initial pure state, we obtain

$$
s(t)=1-\operatorname{Tr}\left(\rho^{2}\right)=\frac{1}{2}\left[1-e^{-2 B^{2}(t)}\right]\left\{\left[\rho_{11}(0)-\rho_{00}(0)\right]^{2}+4\left|\rho_{01}(0)\right|^{2} \sin ^{2}\left[(\Omega / 2) t-\gamma_{0}\right]\right\},
$$

where $\rho_{01}=\left|\rho_{01}\right| e^{-i \gamma_{0}}$.

Note that this result depends on the spectral function $B^{2}(t)$, defined in $(20)$. This function is obtained by integration over the bath mode frequencies. When the summation in (20) is converted to integration in the limit of infinite number of the bath modes, ${ }^{7,13,14}$

$$
B^{2}(t)=8 \int d \omega N(\omega)|g(\omega)|^{2} \omega^{-2} \sin ^{2} \frac{\omega t}{2} \operatorname{coth} \frac{\beta \omega}{2},
$$

where $N(\omega)$ is the density of states. In many realistic models of the bath, the density of states increases as a power of $\omega$ for small frequencies and has a cutoff at large frequencies (Debye cutoff in the case of a phonon bath). Therefore, approximately setting

$$
N(\omega)|g(\omega)|^{2} \propto \omega^{n} \exp \left(-\omega / \omega_{c}\right)
$$

can yield a good qualitative estimate of the relaxation behavior. ${ }^{13,14}$ For a popular case of Ohmic dissipation, ${ }^{12} n=1$, the time dependence of $B^{2}(t)$ is sketched in Figure 2. One can identify the initial stage of quadratic growth of the spectral function, the intermediate region of logarithmic growth, and the linear large-time behavior.

The result (24) illustrates two common features of the decoherence measures. The first is the explicit dependence on the initial density matrix elements. The second is the timedependence that involves the spectral frequencies of the system. We note that the spectral function $B^{2}(t)$ is monotonic. However, the expression (24) also contains oscillatory time dependence with the period $2 \pi / \Omega$, which obviously reflects the property of the system's internal dynamics rather than its deviation from a pure state. If the initial density matrix is diagonal, $\rho_{01}=0$, then we have $s(t)=2^{-1}\left\{1-\left[\rho_{00}(0)-\rho_{11}(0)\right]^{2} e^{-2 B^{2}(t)}\right\}$, which has no such oscillations. 


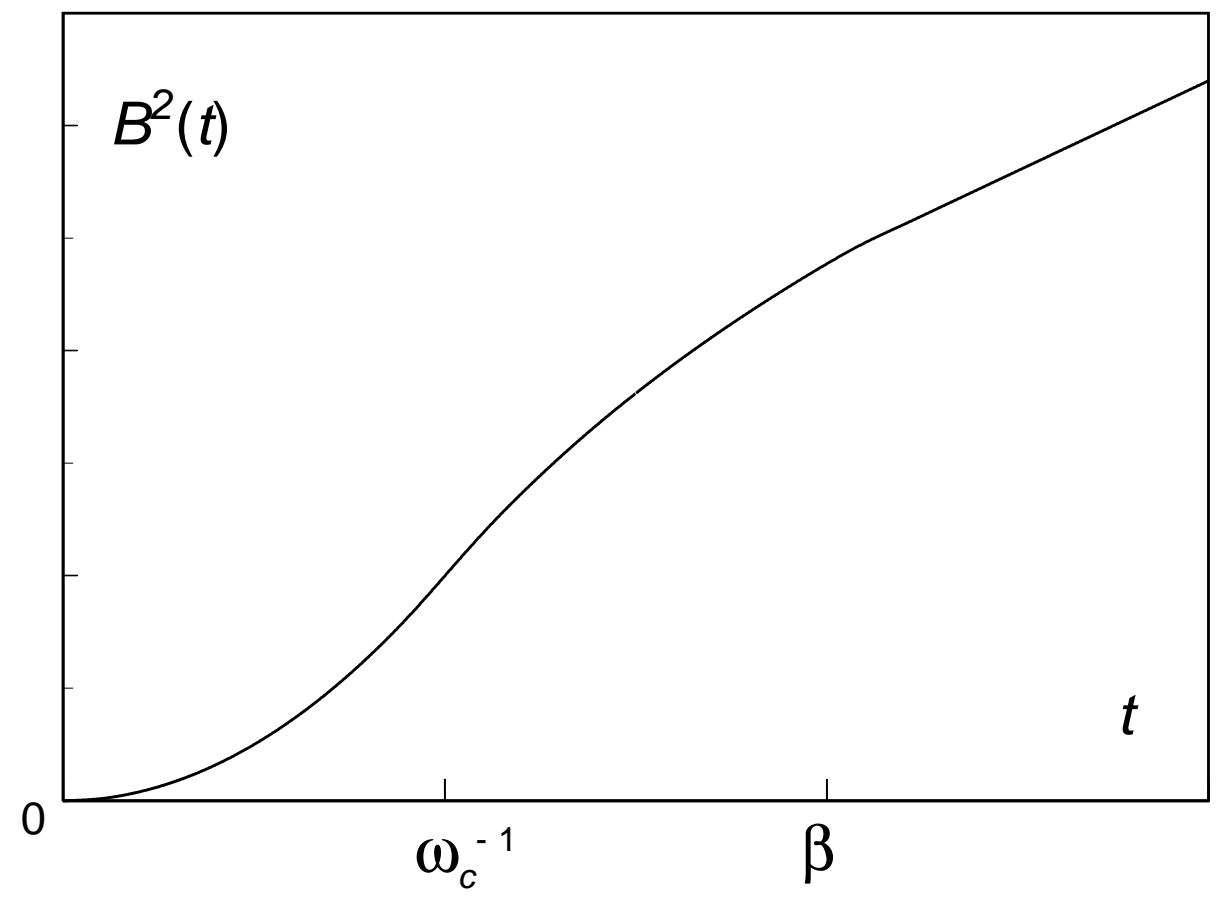

Figure 2: Schematic plot of the spectral function $B^{2}(t)$ for Ohmic dissipation. ${ }^{14,7}$ The "quiet" regime $t<\omega_{c}^{-1}$ corresponds to $B^{2}(t) \propto\left(\omega_{c} t\right)^{2}$, the "quantum" regime $\omega_{c}^{-1}<t<\beta$ corresponds to $B^{2}(t) \propto \ln \left(\omega_{c} t\right)$, and "thermal" regime $t \gg \beta$, corresponds to $B^{2}(t) \propto t / \beta$.

\section{$4 \quad$ Fidelity}

Writing the total Hamiltonian as usual,

$$
H=H_{S}+H_{B}+V
$$

let us now define the fidelity, ${ }^{15,16}$

$$
F(t)=\operatorname{Tr}_{S}\left[\rho_{\text {ideal }}(t) \rho(t)\right]
$$

where the trace is over the system degrees of freedom, and $\rho_{\text {ideal }}(t)$ represents the pure-state evolution of the system under the constant $H_{S}$ only, without interaction with the environment 
$(V=0)$,

$$
\rho_{\text {ideal }}(t)=e^{-i H_{S} t} \rho(0) e^{i H_{S} t} .
$$

The fidelity provides a certain measure of decoherence in terms of the difference between the "real," environmentally influenced, $\rho(t)$, evolution and the "free" evolution, $\rho_{\text {ideal }}(t)$. It will attain its maximal value, 1 , only provided $\rho(t)=\rho_{\text {ideal }}(t)$. This property relies on the fact the $\rho_{\text {ideal }}(t)$ remains a projection operator (pure state) for all times $t \geq 0$.

Let us consider the two-level system decaying from the excited to ground state, $(9,10)$. In this case, there is no internal system dynamics,

$$
\begin{gathered}
\rho_{\text {ideal }}(t)=\left(\begin{array}{ll}
0 & 0 \\
0 & 1
\end{array}\right), \\
\rho(t)=\left(\begin{array}{cc}
1-e^{-\Gamma t} & 0 \\
0 & e^{-\Gamma t}
\end{array}\right),
\end{gathered}
$$

and the fidelity is a monotonic function of time,

$$
F(t)=e^{-\Gamma t} .
$$

Note that the requirement that $\rho_{\text {ideal }}(t)$ is pure-state (projection operator), excludes any $T>0$ thermalized state as the initial system state. For example, let us consider the infinitetemperature initial state of our two level system. We have

$$
\rho(0)=\rho_{\text {ideal }}(t)=\left(\begin{array}{cc}
1 / 2 & 0 \\
0 & 1 / 2
\end{array}\right),
$$

which is not a projection operator. The spontaneuos-decay density matrix is then

$$
\rho(t)=\left(\begin{array}{cc}
1-\left(e^{-\Gamma t} / 2\right) & 0 \\
0 & e^{-\Gamma t} / 2
\end{array}\right) .
$$

The fidelity is constant,

$$
F(t)=1 / 2,
$$

and it does not provide any useful information of the time-dependence of the decay process.

We can derive the short-time perturbative expansion ${ }^{15}$ for $F(t)$,

$$
F(t)=1-\left(t / \tau_{F}\right)^{2}+o\left(t^{2}\right),
$$




$$
1 /\left(2 \tau_{F}^{2}\right)=\left\langle\left\langle V^{2}\right\rangle_{S}\right\rangle_{B}-\left\langle\left(\langle V\rangle_{S}\right)^{2}\right\rangle_{B}
$$

which should be compared to (16).

Let us now turn to the example of the two-level system in the short-time approximation; see (17), et seq. Coherent evolution of the two-level system due to the Hamiltonian $H_{S}$, is described by

$$
\rho_{\text {ideal }}(t)=\left(\begin{array}{cc}
\rho_{00}(0) & \rho_{01}(0) e^{i \Omega t} \\
\rho_{10}(0) e^{-i \Omega t} & \rho_{11}(0)
\end{array}\right) .
$$

A straightforward calculation gives

$$
F(t)=1-\frac{1}{2}\left[1-e^{-B^{2}(t)}\right]\left\{\left[\rho_{11}(0)-\rho_{00}(0)\right]^{2}+4\left|\rho_{01}(0)\right|^{2} \sin ^{2}\left[(\Omega / 2) t-\gamma_{0}\right]\right\}
$$

This result should be compared to (24) While not identical, it has similar features, including the system-frequency oscillations.

\section{Norm of deviation}

In this section we propose to use the operator norms ${ }^{17}$ that measure the deviation of the system from the ideal state, to quantify the degree of decoherence. Such measures do not require the initial density matrix to be pure-state. We define the deviation according to

$$
\sigma(t) \equiv \rho(t)-\rho_{\text {ideal }}(t)
$$

We can use, for instance, the eigenvalue norm,

$$
\|\sigma\|_{\lambda}=\max _{i}\left|\lambda_{i}\right|
$$

or the trace norm,

$$
\|\sigma\|_{\operatorname{Tr}}=\sum_{i}\left|\lambda_{i}\right|
$$

etc., where $\lambda_{i}$ are the eigenvalues of the deviation operator, (40).

For more precise definitions, let us consider an arbitrary linear operator $A$. One of the possible ways to define the norm of $A$ is ${ }^{17}$

$$
\|A\|=\sup _{\varphi \neq 0}\left[\frac{\left\langle\varphi\left|A^{\dagger} A\right| \varphi\right\rangle}{\langle\varphi \mid \varphi\rangle}\right]^{1 / 2} .
$$


Since density operators are bounded, their norms, as well the norm of the deviation, can be always evaluated. Furthermore, since the density operators are Hermitean, this definition obviously reduces to the eigenvalue norm (41). We also note that $\|A\|=0$ implies that $A=0$.

The calculation of these norms is sometimes simplified by the observation that $\sigma(t)$ is traceless. Specifically, for two-level systems, we get

$$
\|\sigma\|_{\lambda}=\sqrt{\left|\sigma_{00}\right|^{2}+\left|\sigma_{01}\right|^{2}}=\frac{1}{2}\|\sigma\|_{\operatorname{Tr}} .
$$

Therefore, for tow-state systems, we will only consider the eigenvalue norm. For our example of the two-level system undergoing spontaneous decay, the norm is

$$
\|\sigma\|_{\lambda}=1-e^{-\Gamma t}
$$

Thus, in this case $\|\sigma\|_{\lambda}=1-F(t)$.

Next, consider the two-level system coupled to a bath of modes, in the short-time approximation; see (17), et seq. The coherent (ideal) evolution of the system due to the Hamiltonian $H_{S}$, is described by (38). One can then obtain the result

$$
\|\sigma(t)\|_{\lambda}=\frac{1}{2}\left[1-e^{-B^{2}(t)}\right]\left\{\left[\rho_{11}(0)-\rho_{00}(0)\right]^{2}+4\left|\rho_{01}(0)\right|^{2} \sin ^{2}\left[(\Omega / 2) t-\gamma_{0}\right]\right\}^{1 / 2}
$$

which should be compared to (24) and (39). At $t=0$, the value of the norm is equal to 0 , and then it increases to positive values, with superimposed modulation at the system's energy-gap frequency.

\section{Arbitrary Initial States, Multiqubit Systems}

The measures considered in the preceding sections quantify decoherence of a system provided its initial state is given. However, this is not always the case. Usually, it will be necessary to obtain an upper-bound estimate of decoherence for an arbitrary initial state. For example, in quantum teleportation schemes, the quantum state to be teleported is not known. In quantum computing, the ideal state of a quantum register is theoretically specified for each step of a quantum algorithm. But from the practical point of view, the bookkeeping becomes intractable even for few-gate algorithms. Furthermore, even the preparation of the initial state can introduce noise.

To characterize decoherence for an arbitrary initial state, pure or mixed, we propose to use the maximal norm, $D$, which is determined as an operator norm maximized over all initial 
density matrices. For instance, we can define

$$
D(t)=\sup _{\rho(0)}\left(\|\sigma(t, \rho(0))\|_{\lambda}\right) .
$$

For the two-level system coupled to a bosonic bath, with the dynamics described by the Hamiltonian (17), the expression of the maximal norm is elegant and compact,

$$
D(t)=\frac{1}{2}\left[1-e^{-B^{2}(t)}\right]
$$

The result is monotonic and contains no oscillations due to the internal system dynamics, as shown in Figure 3.

In principle, the calculation of the maximal norm for a large-scale multiqubit system should be formidable task, since it implies maximization over an exponentially large (in the number of qubits) set of coefficients of the initial density matrix. However, for specific applications, we can use approximate upper bounds calculated by evaluating norms for small subsystems. Consider a multiqubit system $S$ consisting of two separate unentangled subsystems $S_{2}$ and $S_{2}$, at time $t$, with decoherence norms $D_{1}$ and $D_{2}$, respectively. Let us denote the density matrix of the full system and its deviation as $\rho$ and $\sigma$, respectively, and use the same notation with indices 1 and 2 for the two subsystems. For brevity, we use the superscript $(i)$ to denote the "ideal" density matrices. The overall norm $D(t)$ can then be written as

$$
D=\sup _{\rho(0)}\left(\|\sigma\|_{\lambda}\right)=\sup _{\rho(0)}\left(\left\|\rho-\rho^{(i)}\right\|_{\lambda}\right)=\sup _{\rho(0)}\left(\left\|\rho_{1} \rho_{2}-\rho_{1}^{(i)} \rho_{2}^{(i)}\right\|_{\lambda}\right)=\sup _{\rho(0)}\left(\left\|\sigma_{1} \rho_{2}+\rho_{1}^{(i)} \sigma_{2}\right\|_{\lambda}\right)
$$

and estimated by using the following sequence of inequalities,

$$
\begin{gathered}
D=\sup _{\rho(0)}\left(\left\|\sigma_{1} \rho_{2}+\rho_{1}^{(i)} \sigma_{2}\right\|_{\lambda}\right) \leq \sup _{\rho(0)}\left(\left\|\sigma_{1} \rho_{2}\right\|_{\lambda}\right)+\sup _{\rho(0)}\left(\left\|\rho_{1}^{(i)} \sigma_{2}\right\|_{\lambda}\right), \\
\sup _{\rho(0)}\left(\left\|\sigma_{1} \rho_{2}\right\|_{\lambda}\right)+\sup _{\rho(0)}\left(\left\|\rho_{1}^{(i)} \sigma_{2}\right\|_{\lambda}\right) \leq \sup _{\rho_{1}(0)}\left(\left\|\sigma_{1}\right\|_{\lambda}\right)+\sup _{\rho_{2}(0)}\left(\left\|\sigma_{2}\right\|_{\lambda}\right)=D_{1}+D_{2} .
\end{gathered}
$$

The inequality $D \leq D_{1}+D_{2}$ is, of course, approximate, because we have assumed that the interaction with the bath, and with each other, left the subsystems unentangled at time $t$. However, it provides an indication that, perhaps with a smart choice of a decomposition into subsystems which are initially not significantly entangled, the maximal norm can be approximately considered an extensive quantity, linear in the number of qubits, at least for short times. It is expected that for larger times, the relaxation process rates are the additive 


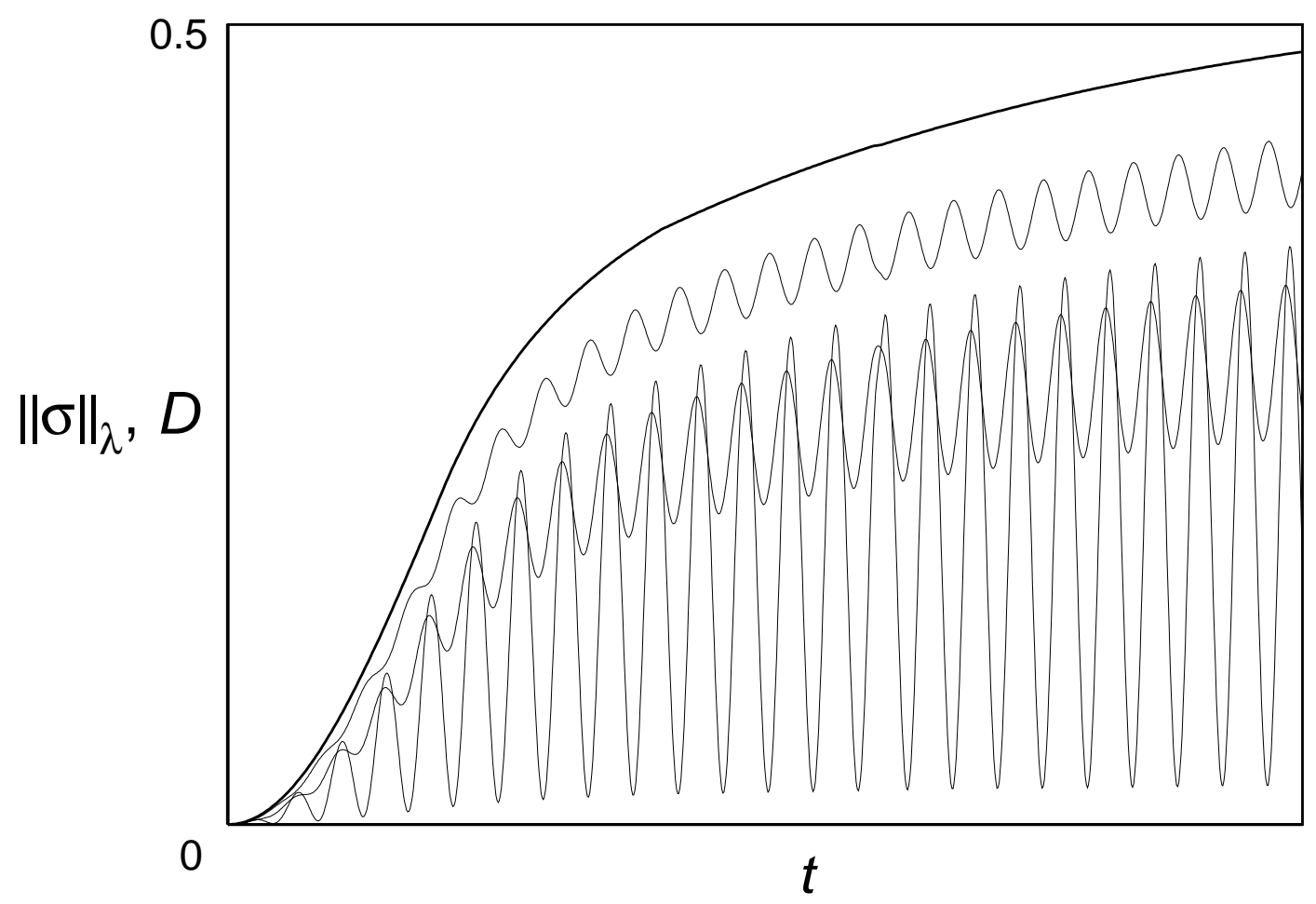

Figure 3: Norm of the deviation from the ideal dynamics for the density matrix of a spin interacting with an Ohmic bath of bosonic modes, in the short-time approximation. The upper curve: the maximal norm $D(t)$; the lower curves: the $\|\sigma\|_{\lambda}$ norms for three different initial states $\rho(0)$, chosen to illustrate the overall pattern.

quantities growing linearly with the number of qubits, as long as no quantum error correction is involved.

In conclusion, we have considered several approaches to quantify decoherence: relaxation times, entropy and fidelity measures, and norms of deviation. The latter measures offer certain advantages, and we defined the maximal measure that is not dependent on the initial state and, at least for short times, is approximately extensive in the number of qubits.

This research was supported by the National Security Agency and Advanced Research and Development Activity under Army Research Office contract DAAD-19-02-1-0035, and by the National Science Foundation, grants DMR-0121146 and ECS-0102500. 


\section{REFERENCES}

[1] D. Aharonov and M. Ben-Or, e-prints quant-ph/9611025 and quant-phys/9906129 (at www.arxiv.org).

[2] A. Abragam, The Principles of Nuclear Magnetism (Oxford University Press, 1961).

[3] K. Blum, Density Matrix Theory and Applications (Plenum, New York, 1996).

[4] N. G. van Kampen, Stochastic Processes in Physics and Chemistry (North-Holland, Amsterdam, 1992).

[5] D. P. DiVincenzo, e-print quant-ph/0002077 (at www.arxiv.org).

[6] V. Privman, J. Stat. Phys. 110, 957 (2003).

[7] D. Mozyrsky and V. Privman, J. Stat. Phys. 91787 (1998).

[8] J. von Neumann, Mathematical Foundations of Quantum Mechanics (Princeton University Press, 1983).

[9] J. I. Kim, M. C. Nemes, A. F. R. de Toledo Piza and H. E. Borges, Phys. Rev. Lett. 77, 207 (1996).

[10] W. H. Zurek, S. Habib and J. P. Paz, Phys. Rev. Lett. 70, 1187 (1993).

[11] J. C. Retamal and N. Zagury, Phys. Rev. A 63, 032106 (2001).

[12] A. J. Leggett, S. Chakravarty, A. T. Dorsey, M. P. A. Fisher, A. Garg and W. Zwerger, Rev. Mod. Phys. 59, 1 (1987).

[13] N. G. van Kampen, J. Stat. Phys. 78, 299 (1995).

[14] G. M. Palma, K. A. Suominen, A. K. Ekert, Proc. Roy. Soc. Lond. A 452, 567 (1996).

[15] B. J. Dalton, e-print quant-ph/0209071 (at www.arxiv.org).

[16] L.-M. Duan and G.-C. Guo, Phys. Rev. A 56, 4466 (1997).

[17] T. Kato, Perturbation Theory for Linear Operators (Springer-Verlag, New York, 1995). 